\title{
Genus Promalactis Meyrick (Lepidoptera: Oecophoridae) in northern Vietnam, Part III: seven new species and four newly recorded species
}

\author{
Sora Kim ${ }^{\mathrm{a}}$, Kyu-Tek Park ${ }^{\mathrm{b}}$, John B. Heppner ${ }^{\mathrm{b}}$ and Seunghwan Lee ${ }^{\mathrm{a} *}$ \\ ${ }^{a}$ Division of Entomology, School of Agricultural Biotechnology, Research Institute for \\ Agriculture and Life Sciences, Seoul National University, Seoul, Korea; ${ }^{b}$ McGuire Center for \\ Lepidoptera and Biodiversity, Florida Museum of Natural History, University of Florida, \\ Gainesville, FL, USA
}

(Received 28 January 2013; accepted 24 October 2013; first published online 3 March 2014)

\begin{abstract}
This article is the third part of a series on the genus Promalactis (Lepidoptera: Oecophoridae) in northern Vietnam. Seven new species, Promalactis acanthiana Kim and Park, sp. nov., Promalactis albinparata Kim and Park, sp. nov., Promalactis asperiola Kim and Park, sp. nov., Promalactis caperatiella Kim and Park, sp. nov., Promalactis curtifasciata Kim and Park, sp. nov., Promalactis gigaspinata Kim and Park, sp. nov. and Promalactis reniformis Kim and Park, sp. nov. are described, and four species, Promalactis basifasciaria Wang, Promalactis kalimantana Lvovsky, Promalactis tauricournis Du, Li and Wang and Promalactis tricuspidata Wang and $\mathrm{Li}$ are reported for the first time from Vietnam. Illustrations of adults and genitalia of the species are provided.
\end{abstract}

http://zoobank.org/urn:lsid:zoobank.org:pub:75F7CC58-F8CD-4B50-A0DAD0D6544AA65A

Keywords: Oecophoridae; Promalactis; new species; Vietnam, taxonomy

\section{Introduction}

The genus Promalactis Meyrick of the family Oecophoridae (Lepidoptera, Gelechioidea) is one of the most diverse genera of the family. The genus is distributed primarily in the Oriental and Palaearctic Regions, comprising more than 160 species (Meyrick 1908a, 1908b; Lvovsky 1976, 1985, 1986, 1988, 1997, 2000a, 2000b; Park 1981; Park and Park 1998; Fujisawa 2002; Wang 2006; Du et al. 2009; Wang et al. 2009; Kim et al. 2010, 2012; Du et al. 2011). However, it is assumed that the actual number might be more than twice the known species.

Promalactis species are superficially characterized by the brownish-yellow to yellowish-brown forewing ground colour with variable markings, and the wing venation with radius IV and radius $\mathrm{V}$ stalked.

So far, more than 40 species have been recognized from the Oriental Region including India, Indonesia, Malaysia, Myanmar, Philippines and Vietnam (Meyrick 1908a, 1908b; Lvovsky 1988, 1997, 2000a, 2000b, 2007; Kim et al. 2010, 2012). In Vietnam, 19 species of the genus are known (Lvovsky 1988, 1997, 2007; Kim et al. 2010, 2012).

*Corresponding author. Email: seung@snu.ac.kr 


\title{
Material and methods
}

Material examined for the present study is based on specimens collected in three different national parks, Ba Be (1080 m), Cuc Phuong (155 m, $196 \mathrm{~m}$ and $200 \mathrm{~m})$, and Tam Dao (1108 m, $1000 \mathrm{~m}$ and $950 \mathrm{~m}$ ) in the northern part of Vietnam, from April to August during 2005-2009 by a sweeping net during daytime, and by light traps and bucket traps at night (from 7-8 p.m. to 1 a.m.).

Specimens were examined under a microscope and photographs were taken from digital images using the software, Image Lab version 2.2.4.0 by MCM Design (Hillerød, Denmark). The colour standard for the description of adults follows Kornerup and Wanscher (1978). All types are deposited in the McGuire Center for Lepidoptera and Biodiversity, Florida Museum of Natural History, University of Florida, USA, on indefinite loan from Vietnam.

\section{Systematic accounts}

\author{
Genus Promalactis Meyrick, 1908
}

Promalactis Meyrick, 1908a, J. Bombay Nat. His. Soc., 18: 806.

Type species: Promalactis holozona Meyrick, 1908. Type locality: Coorg, S. India. Promalactis acanthiana Kim and Park, sp. nov.

(Figures 1A, B, 2A, and 3A-D)

\section{Diagnosis}

This species is externally similar to Promalactis albisquama Kim and Park, and Promalactis clavata Du, Li and Wang, by having an oblique band-like marking and a semi-circular costal patch in forewing pattern, but can be distinguished by the male genitalia with a long cornutus of the aedeagus.

\section{Description}

Adult (Figure 1A, B). Head (Figure 2A): Frons shiny whitish-yellow, vertex deep yellowish-brown. Antenna with scape entirely white, as long as diameter of eye; flagellum white basally and mixed with dark brown alternately from one-third along to apex. Labial palpus dark brown except basal part of third segment entirely white; third segment almost same length as second segment. Thorax: Thorax yellowish-brown; tegula darker than thorax. Wing expanse 8.0-8.5 mm. Forewing ground colour yellowish-brown; two white markings surrounded by fuscous scales presented; one oblique, from one-third costal margin transverse to one-half of posterior margin, band-like and the other costal patch at two-thirds of costa of forewing; fringes white at termen, speckled with fuscous scales, partly greyish-brown fringes beyond apex and before tornus. Hindwing more or less lanceolate; fringes greyish-brown. Leg: hind leg pale yellowish-brown ventrally, except dark brown medial to fifth segment of tarsus.

Male genitalia (Figure 3A-D). Uncus bell-shaped, wide at base. Gnathos tongue-like, as long as uncus. Valva more or less broad; costa densely setose from one-third beyond apex to apical sacculus; apex thumb-like. Juxta with a pair of symmetrical lobes, extremely long, slightly shorter than valva. Saccus wide at base, as long as 


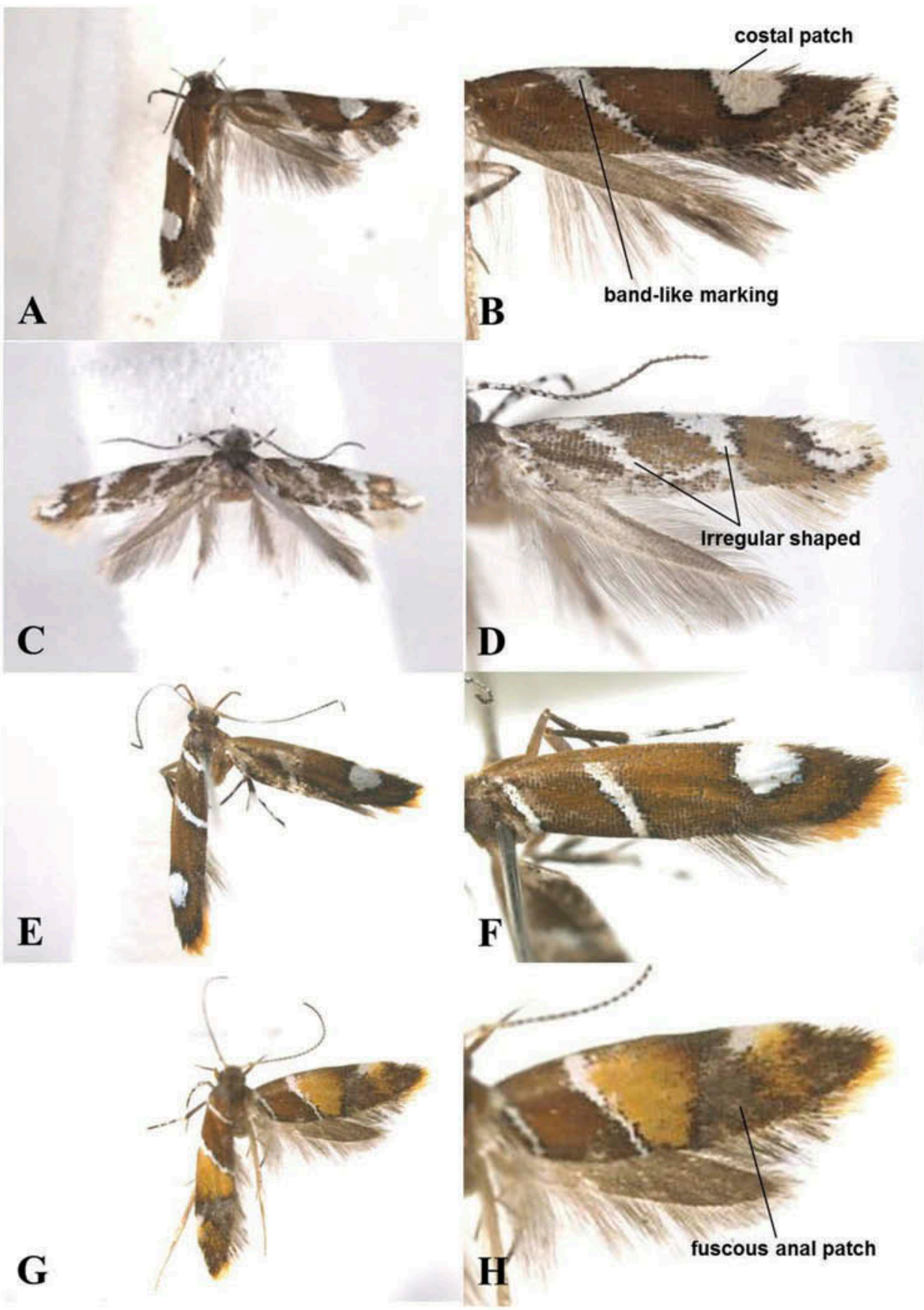

Figure 1-1. Adult. (A) Promalactis acanthiana sp. nov.; (B) Promalactis acanthiana sp. nov., wing pattern; (C) Promalactis albinparata sp. nov.; (D) Promalactis albinparata sp. nov., wing pattern; (E) Promalactis asperiola sp. nov.; (F) Promalactis asperiola, wing pattern; (G) Promalactis. basifasciaria; $(\mathrm{H})$ Promalactis. basifasciaria, wing pattern. 

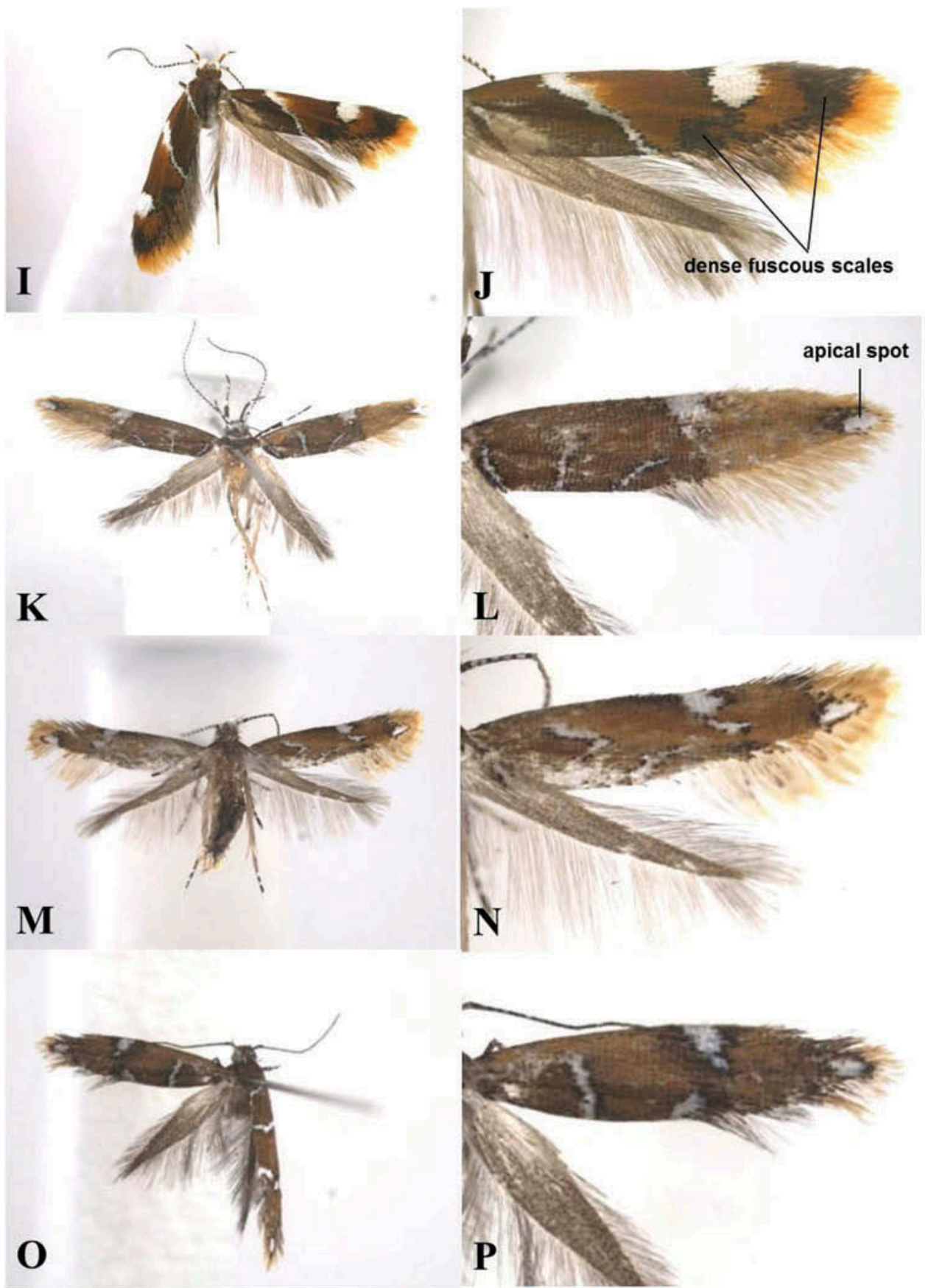

Figure 1-2. Adult. (I) Promalactis caperatiella sp. nov.; (J) Promalactis caperatiella sp. nov., wing pattern; (K) Promalactis curtifascia sp. nov.; (L) Promalactis curtifascia sp. nov., wing pattern; (M) Promalactis gigaspina sp. nov.; (N) Promalactis gigaspina sp. nov., wing pattern; (O) Promalactis kalimantana; (P) Promalactis kalimantana, wing pattern. 


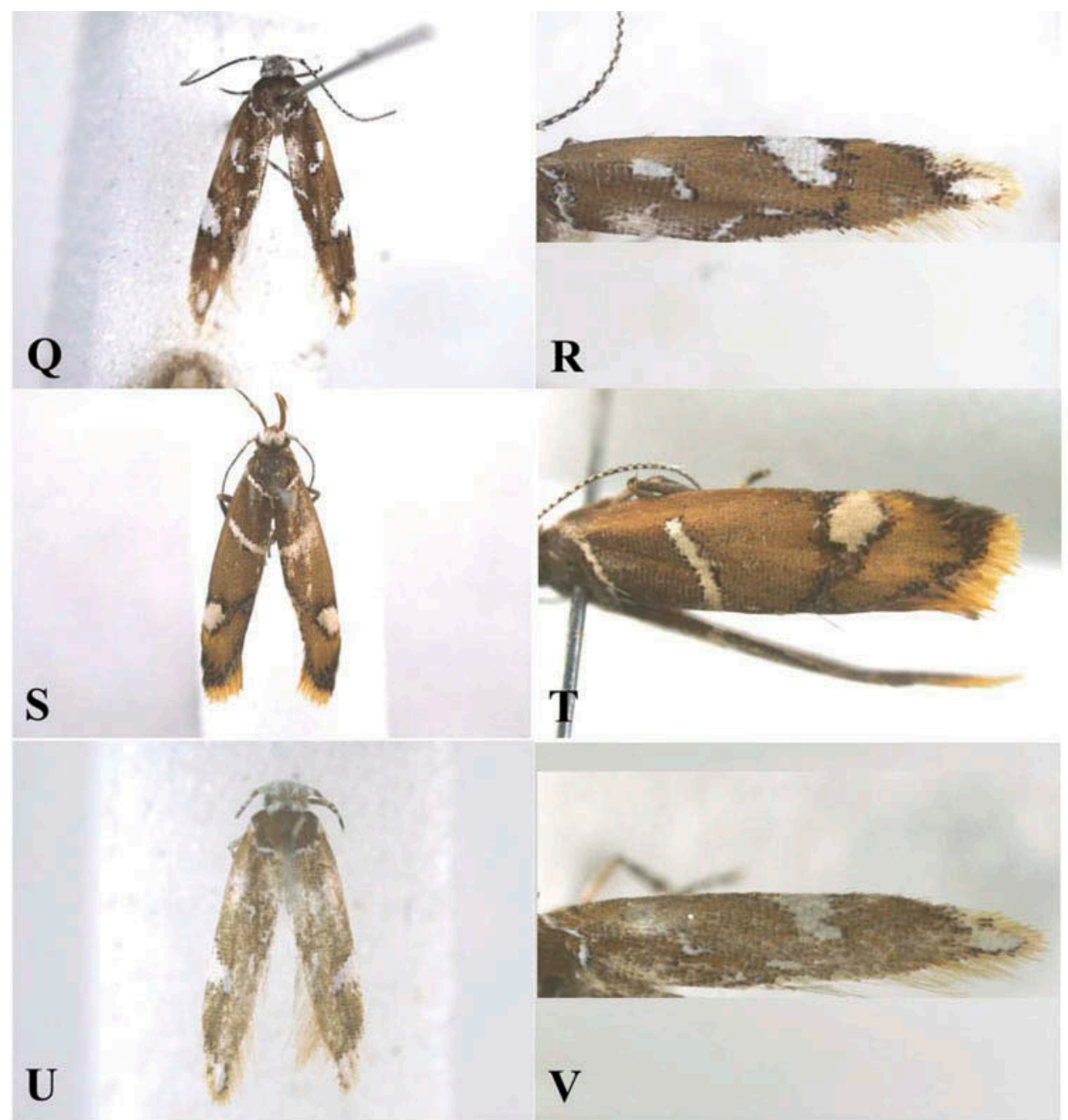

Figure 1-3. Adult. (Q) Promalactis reniformis; (R) Promalactis reniformis, wing pattern; (S) Promalactis tauricournis; (T) Promalactis tauricournis, wing pattern; (U) Promalactis tricuspidata; (V) Promalactis tricuspidata, wing pattern.

tegumen. Aedeagus long, straightly developed, as same length as juxta; cornutus placed apically, one-quarter length of aedeagus.

Female genitalia. Unknown.

\section{Holotype}

Male, Tam Dao Nat. Park, Vietnam, 1000 m, 5 May 2005, KT Park and S Kim, slide gen. no. SNU-9273/S Kim; Paratype. One male, Tam Dao Nat. Park, 


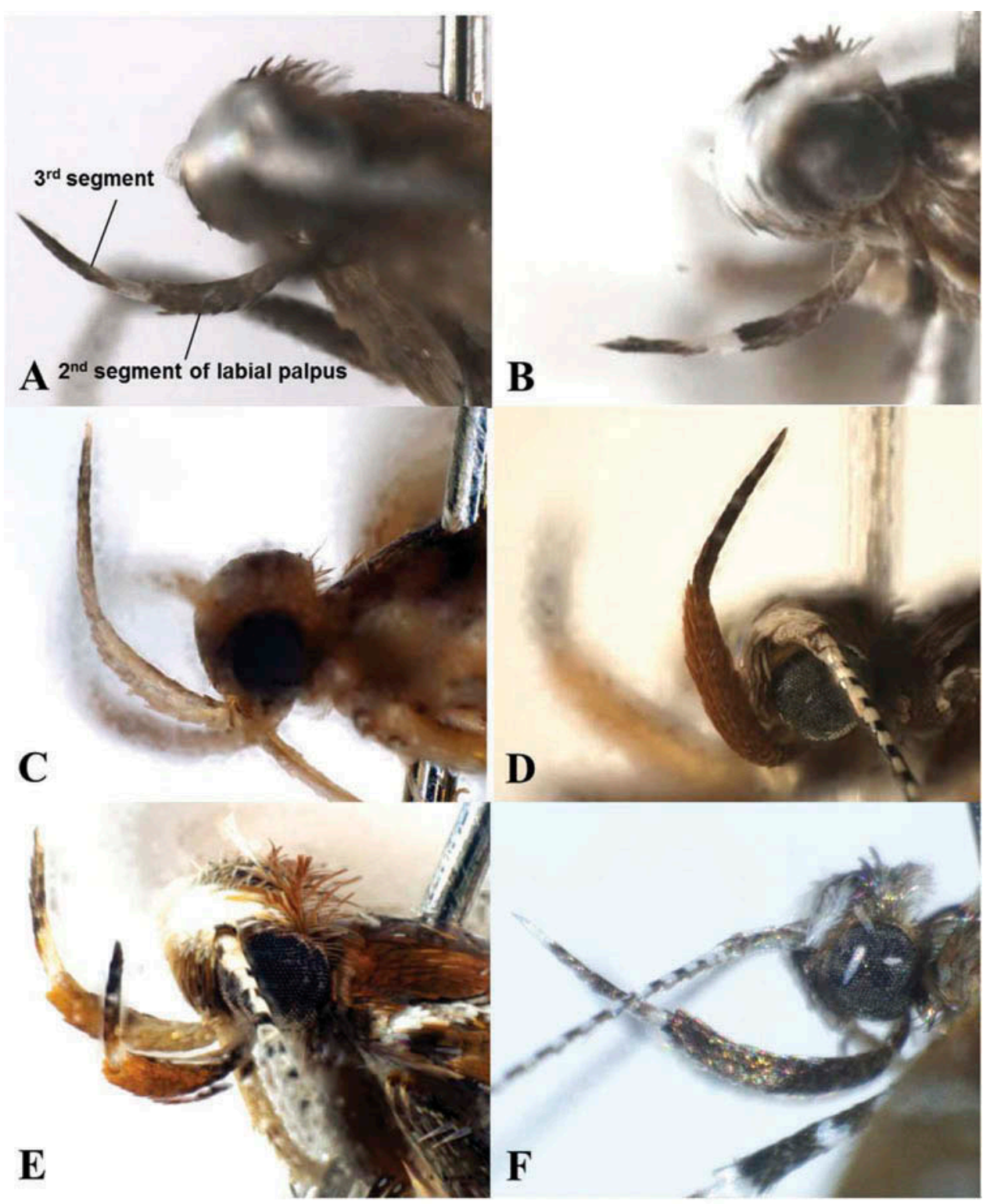

Figure 2-1. Head. (A) Promalactis acanthiana sp. nov.; (B) Promalactis albinparata sp. nov.; (C) Promalactis asperiola sp. nov.; (D) Promalactis basifasciaria; (E) Promalactis caperatiella sp. nov.; (F) Promalactis curtifasciata sp. nov.

Vietnam, 1000 m, 4 May 2005, KT Park and S Kim, slide gen. no. SNU-9276/ S Kim.

\section{Distribution}

Oriental: Vietnam (North). 

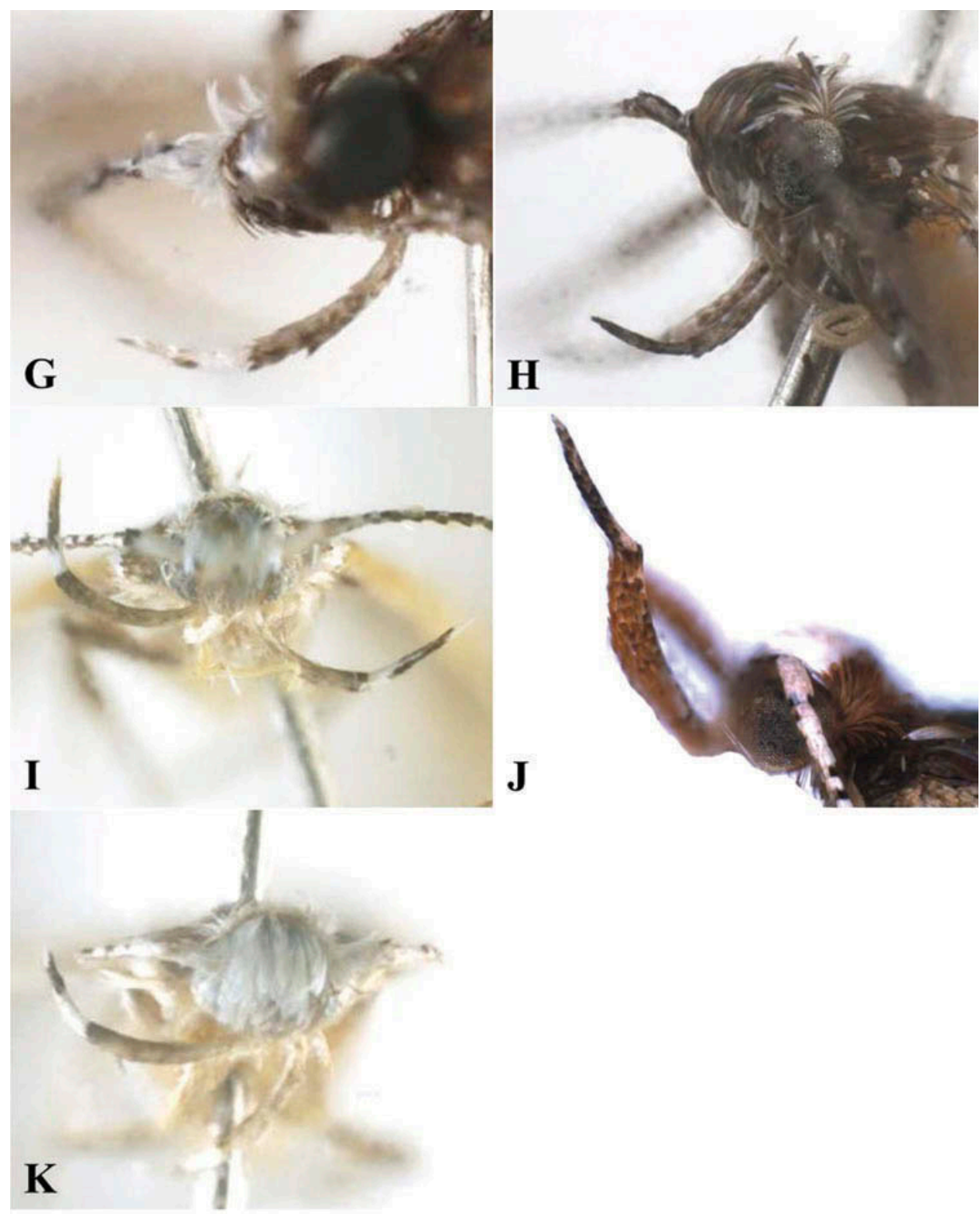

Figure 2-2. Head. (G) Promalactis gigaspinata sp. nov.; (H) Promalactis kalimantana; (I) Promalactis reniformis; (J) Promalactis tauricournis; (K) Promalactis tricuspidata sp. nov.

Etymology

The specific name is derived from the Latin, acanth $-i$ (= spine) plus a Latin suffix -ana, referring to spine-like cornutus of the male genitalia.

Promalactis albinparata Kim and Park, sp. nov.

(Figures 1C, D, 2B, and 3E, F) 

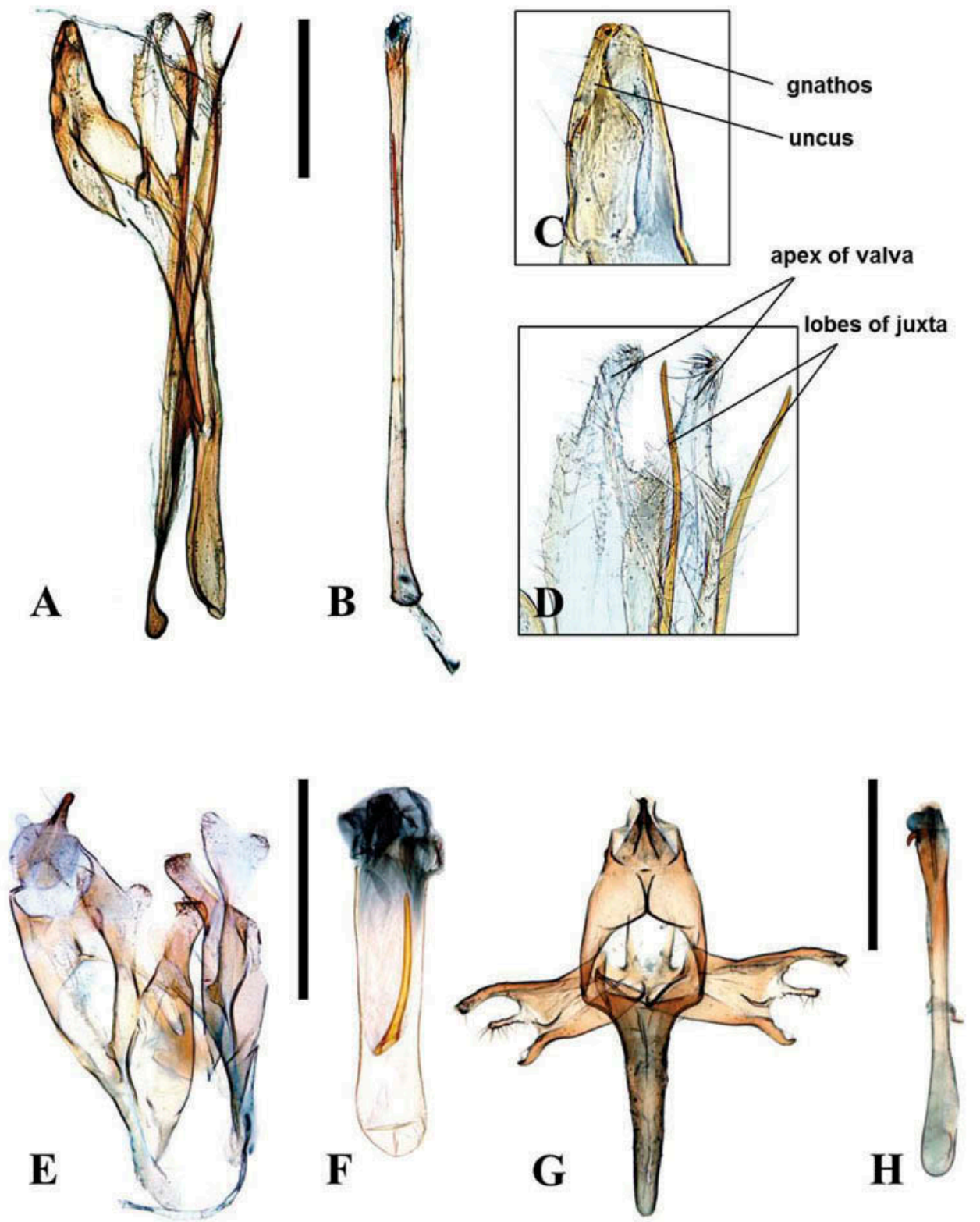

Figure 3. Male genitalia. (A) Promalactis acanthiana sp. nov.; (B) aedeagus; (C) gnathos and uncus; (D) apical part of valva; (E) Promalactis albinparata sp. nov.; (F) Promalactis albinparata sp. nov., aedeagus; (G) Promalactis tricuspidata; (H) Promalactis tricuspidata, aedeagus. Scale bar $0.5 \mathrm{~mm}$.

\section{Diagnosis}

This species is superficially similar to Promalactis jezonica (Matsumura) in the wing pattern, but can be distinguished by the irregularly shaped white markings and fuscous scales crossing the apical spot of the forewing. 


\section{Description}

Adult (Figure 1C, D). Head (Figure 2B): Frons white; vertex dark brown. Antenna with white scape entirely, as long as diameter of eye; flagellum white and dark brown alternately. Labial palpus dark brown, except white apical and basal part of third segment; second segment 1.5 times longer than third segment. Thorax: Thorax and tegula dark brown dorsally. Wing expanse $7.0-7.5 \mathrm{~mm}$. Forewing ground colour dark brown; two white bands oblique surrounded by fuscous scales: one near to wing base, the other at one-sixth of costal margin, not reaching to posterior margin; one white costal patch at two-thirds, somewhat triangular-shaped, surrounded by fuscous scales; another white apical patch surrounded and crossed by fuscous scales, except outer margin above apex; two white short streaks rarely surrounded by fuscous scales under medial vein: one between second band and costal patch, the other one under costal patch; fringes yellowishbrown, tinged with greyish-brown from apex to half of outer margin. Hindwing more or less lanceolate; fringes greyish-brown. Legs: hind leg pale yellowishbrown, except apical parts of fifth and third segments and basal parts of first and second segment of tarsus.

Male genitalia (Figure $3 E, F$ ). Uncus bell-like, wide at base, gradually narrowed to apex; caudal margin of apex sclerotized. Gnathos thumb-like, wide at base, shorter than uncus. Valva symmetric; cucullus clavate with sclerotized, setose, flattened apex; processes of sacculus wide to apex, caudal margin of apex incised medially and setose. Aedeagus cylindrical, wide, shorter than sacculus; cornutus about half length of aedeagus.

Female genitalia. Unknown.

\section{Holotype}

One male, Cuc Phuong Nat. Park, Vietnam, 200 m, 1-2 July 2008, JB Heppner, slide gen. no. SNU-9334/S Kim. Paratype. One male, same locality, 1-2 July 2008, JB Heppner, slide gen. no. SNU-9052/S Kim.

\section{Etymology}

The specific name is derived from the Latin, alb- (= white) plus inpar (= irregular or uneven), referring to white irregular-shaped markings of the forewing.

\section{Distribution}

Oriental: Vietnam (North).

Promalactis asperiola Kim and Park, sp. nov.

(Figures 1E, F, 2C, and 4A-C) 

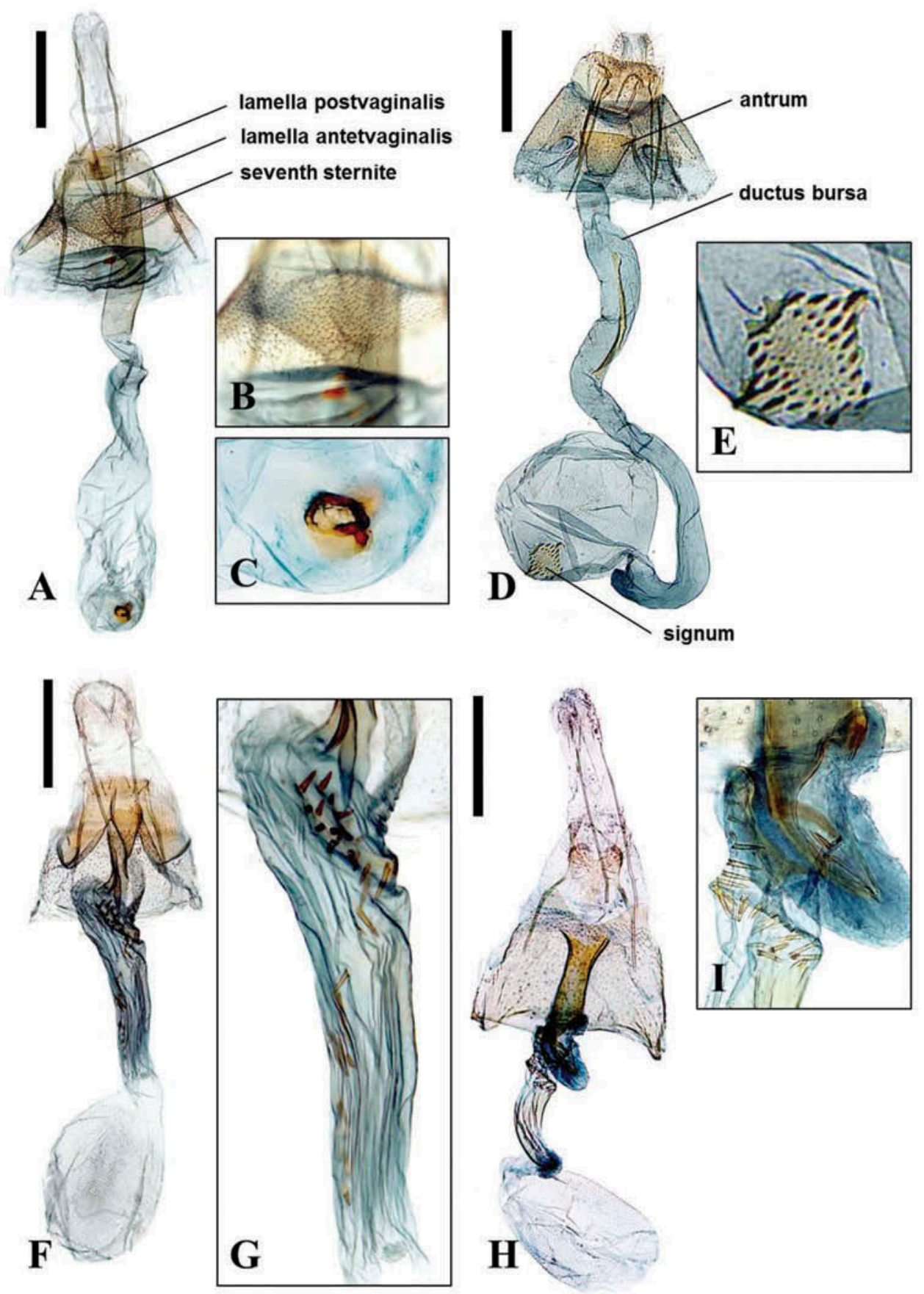

Figure 4-1. Female genitalia. (A) Promalactis asperiola sp. nov.; (B) Promalactis asperiola sp. nov.; surface of seventh sternite in detail; (C) Promalactis asperiola sp. nov., signum; (D) Promalactis basifasciaria; (E) Promalactis basifasciaria, signum; (F) Promalactis caperatiella sp. nov.; (G) Promalactis caperatiella sp. nov., signum; (H) Promalactis curtifasciata sp. nov.; (I) Promalactis curtifasciata sp. nov., ductus bursa in detail. Scale bar $0.5 \mathrm{~mm}$. 

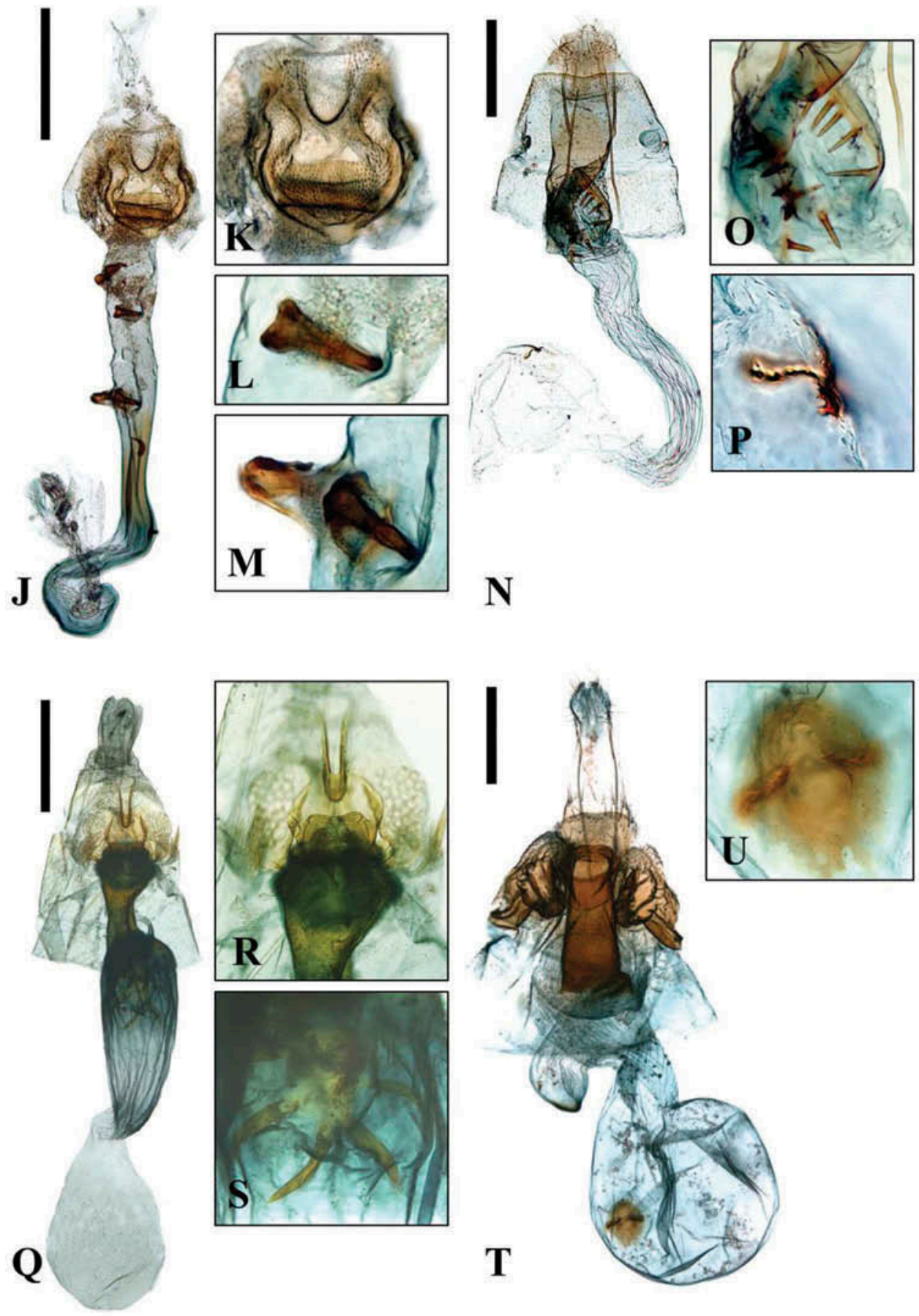

Figure 4-2. Female genitalia. (J) Promalactis gigaspinata sp. nov.; (K) Promalactis gigaspinata sp. nov., lamella antevaginalis; (L) Promalactis gigaspinata sp. nov., basal spine of ductus bursa; (M) Promalactis gigaspinata sp. nov., medial spine of ductus bursa; (N) Promalactis kalimantana; (O) Promalactis kalimantana, basal part of ductus bursa; (P) Promalactis kalimantana; signum; (Q) Promalactis reniformis sp. nov.; (R) Promalactis reniformis sp. nov., lamella antevaginalis and antrum; (S) Promalactis reniformis sp. nov., basal spines of ductus bursa; (T) Promalactis tauricournis; (U) Promalactis tauricournis signum. Scale bar $0.5 \mathrm{~mm}$. 


\section{Diagnosis}

This species is similar to Promalactis suzukiella (Matsumura) in the wing pattern, but can be distinguished by the rough surface of seventh sternite and a ring-shaped signum in the female genitalia.

\section{Description}

Adult (Figure 1E, F). Head (Figure 2C): Frons greyish-brown; vertex dark brown. Antenna with scape white entirely; flagellum white dorsally. Labial palpus dark brown, except second segment, which is yellowish-brown ventrally; third segment same length as second segment. Thorax: Thorax and tegula yellowish-brown dorsally. Wing expanse 7.5-8.0 mm. Forewing ground colour yellowish-brown. White markings surrounded by fuscous scales, except apical margin; two oblique bands, one at near base, the other from one-third costal margin transverse to posterior margin, another white costal patch at two-thirds of forewing; two fuscous patches, one under costal patch mixed with pale grey, the other at subapical margin of forewing; fringes yellow from apex to tornus, followed by the outer margin, tinged with dark-brown scales near tornus. Hindwing more or less lanceolate; fringes greyish-brown. Legs: hind leg dark brown ventrally, except apical and basal parts of fifth, basal part of fourth and first segment of tarsus white.

Male genitalia. Unknown.

Female genitalia. (Figure 4A-C). Apophysis posterioris more than 2.5 times length of apophysis anterioris. Lamella postvaginalis incised medially on caudal margin with setose. Lamella antevaginalis incised medially on saw-like caudal margin. Surface of seventh sternite not smooth (Figure 4B). Antrum funnel-shaped, almost same length as papilla anales. Ductus bursae twisted once at two-thirds width. Corpus bursae with a doughnut-like signum.

\section{Holotype}

Female, Ba Be Nat. Park, Vietnam, 26-28 July 2006, Park, Chae and Cuong, slide gen. no. SNU-9047/S Kim. Paratype. One female, same locality, 26-28 July 2006, Park, Chae and Cuong, slide gen. no. SNU-9046/S Kim.

\section{Etymology}

The specific name of the new species is derived from the Latin, asper $-i$ (=rough) plus -ola (a Latin diminutive suffix), referring to rough surface of seventh sternite of female genitalia.

\section{Distribution}

Oriental: Vietnam (North). 
Promalactis basifasciaria Wang 2006

(Figures 1G, H, 2D, and 4D, E)

Promalactis basifasciaria Wang 2006, Science press, 19-20.

\section{Diagnosis}

This species is externally close to Promalactis autoclina Meyrick in the wing pattern, but it is differentiated by the fuscous patch before the tornus in the forewing, the antrum trapezoidal, the duscus bursae with a long spine, and the corpus bursae with diamond-like signum in the female genitalia.

\section{Material examined}

One male, Tam Dao Nat. Park, Vietnam, 950 m, 31 July 2006, Park, Chae and Cuong; one female, same locality, 450 m, 30 July 2006, Park, Chae and Cuong; one female, same locality, 950 m, 24 May 2007, KT Park, slide gen. no. SNU-9004/S Kim.

\section{Distribution}

Oriental: Vietnam (North), China (Southwest; Wang 2006).

Promalactis caperatiella Kim and Park, sp. nov.

(Figures 1I, J, 2E, and 4F, G)

\section{Diagnosis}

This species is similar to P. suzukiella (Matsumura) in the wing marking, but it can be easily differentiated by the presence of fuscous scales under the white costal patch before the tornus.

\section{Description}

Adult (Figure 1I, J). Head (Figure 2E): Frons greyish-brown; vertex white. Antenna with white scape entirely, as long as diameter of eye; flagellum white and dark brown alternately. second segment of labial palpus yellowish-brown; third segment dark brown entirely, except white basal part; third segment shorter than second segment. Thorax: Thorax and tegula dark brown dorsally. Wing expanse 7.0-7.5 mm. Forewing ground colour dark brown at basal and apical parts, and yellow at middle part. White marking surrounded by fuscous scales: two oblique bands presented: one near base, the other one-third from costal margin transverse to one half posterior margin; one semi-circular costal patch at two-thirds; two fuscous patches: one before tornus; the other before apex; fringes yellow followed by the outer margin, dark brown dense before the tornus. Hindwing more or less lanceolate; fringes greyishbrown. Legs: hind leg dark brown dorsally. 
Female genitalia (Figure 4F, G). Papilla anales setose. Apophysis posterioris more than 1.5 times length of apophysis anterioris. Lamella postvaginalis weakly developed. Lamella antevaginalis marginally folded on seventh sternite. Antrum tulipshaped, longer than papilla anales. Ductus bursae broad, wrinkled vertically, spinous at basal and lateral margin. Corpus bursae pear-like, without a signum.

\section{Holotype}

Female, Tam Dao Nat. Park, Vietnam, 950 m, 26 April 2006, Park, Kim and Kang, slide gen. no. SNU- 9045/S Kim. Paratypes. One female, Tam Dao Nat. Park, Vietnam, 950 m, 31 July 2006, Park, Chae and Cuong, slide gen. no. SNU-9268/S Kim; one female, same locality, 24 May 2007, slide gen. no. SNU-9006/S Kim.

\section{Etymology}

The specific name is derived from the Latin, caperat $-i$ (= wrinkled) plus -ella (a Latin diminutive suffix), referring to distinctly wrinkled ductus bursa of female genitalia.

\section{Distribution \\ Oriental: Vietnam (North).}

Promalactis curtifasciata Kim and Park, sp. nov. (Figures 1K, L, 2F, and 4H, I)

\section{Diagnosis}

This species is externally close to Promalactis odaiensis Park in the wing pattern, but it can be easily differentiated by the dark brown ground colour, from wing base to three-fifths of length, and tinged with yellow to apex in forewing and the absence of signum in the female genitalia.

\section{Description}

Adult (Figure 1K, L). Head (Figure 2F): Frons and vertex dark brown. Scape of antenna entirely white, except dark brown laterally, as long as diameter of eye; flagellum white and dark brown alternately. Labial palpus dark brown entirely, except white at basal and apical parts of third segment; second segment 1.5 times longer than third segment. Thorax: Thorax and tegula brown dorsally. Wing expanse 9.0-9.5 mm. Forewing ground colour dark brown from wing base to three-fifths of length, tinged with yellow to apex; white markings: three short streaks from posterior margin and surrounded by fuscous scales: first at near base, second at one-sixth, third at two-sixths; one costal patch at middle; one apical spot after dense fuscous scales; fringes yellow beyond costal patch to apex followed by outer margin, dense and greyish-brown at half of posterior margin. Hindwing lanceolate; fringes greyishbrown. Legs: hind tibia pale yellowish-brown; tarsus pale yellowish-brown, but white at basal and apical parts of fifth, basal part of fourth and third segments. 
Male genitalia. Unknown.

Female genitalia (Figure 4H, I). Apophysis posterioris twice as long as the apophysis anterioris. Lamella postvaginalis largely developed, incised at middle and setose. Lamella antevaginalis deeply incised, "U"-shaped. Antrum same length as papillae analis. Ductus bursae coiled, spinous anteriorly, vertical lined from two-thirds to corpus bursae. Corpus bursae pear-shaped, without signum.

\section{Holotype}

One female, Ba Be, Vietnam, 1080 m, 19-20 July 2010, JB Heppner, slide gen. no. SNU-9338/S Kim.

\section{Etymology}

The specific name of the new species is derived from the Latin, curti (= short) plus fascia $(=$ band), referring to three short band-like markings on the forewing.

\section{Distribution \\ Oriental: Vietnam (North).}

Promalactis gigaspinata Kim and Park, sp. nov. (Figures 1M, N, 2G, and 4J-M)

\section{Diagnosis}

This species is differentiated from congeners by having two white markings at twothirds length of the forewing and the distinct lamella antevaginalis in the female genitalia.

\section{Description}

Adult (Figure 1M, N). Head (Figure 2G): Frons white; vertex greyish-brown. Antenna with entirely white scape, as long as diameter of eye; flagellum white and dark brown alternately. Labial palpus pale yellowish-brown ventrally; second segment dark brown dorsally, longer than third segment; third segment white dorsally except basal and apical part dark brown. Thorax: Thorax and tegula dark brown dorsally. Wing expanse 6.0-6.5 mm. Forewing ground colour yellowish-brown; white markings surrounded by fuscous scales: one oblique band near at base, two short streaks oblique in the opposite direction and connected at one-quarter; one short streak from one-third posterior margin, oblique; one costal patch at two-thirds, the other short streak under the costal patch; one apical spot somewhat triangular-form; fuscous scales dense before the costal patch and tornus; fringes yellow from four-fifths costal margin to tornus, partly mixed with fuscous scales on vein radius IV and before tornus. Hindwing lanceolate; fringes greyish-brown. Leg: hind tibia pale-yellowishbrown with pale-yellowish-brown fringes, apical spurs more than twice as long as 


\section{S. Kim et al.}

basal spurs; tarsus of hind leg dark brown except white at basal parts of fifth, fourth and third and apical part of second segment.

Female genitalia (Figure $4 J-M$ ). Papilla anales missing. Apophysis posterioris and anterioris absent. Ostium deeply incised, U-like. Lamella antevaginalis distinct, setose and roundly margined as in Figure 4K. Ductus bursae almost five times longer than seventh sternite, bearing six spines, gradually narrowed to three-fifths and coiled at four-fifths. Corpus bursae weakly developed without signum.

\section{Holotype}

One female, Tam Dao Nat. Park, Vietnam, 750 m, 15 August 2005, KT Park, MY Kim and MY Chae, slide gen. no. SNU-9286/S Kim.

\section{Etymology}

The specific name of the new species is derived from the Latin, giga (= giant) plus spina (= spine), referring to large spines in female genitalia.

\section{Distribution \\ Oriental: Vietnam (North).}

Promalactis kalimantana Lvovsky, 2000

(Figures 1O, P, 2H, and 4N-P)

\section{Diagnosis}

This species is similar to Promalactis thiasitis Meyrick in the wing pattern, but can be distinguished by the ductus bursae bearing spines basally, and the corpus bursae with distinct signum in the female genitalia.

\section{Material examined}

One female, Cuc Phuong Nat. Park, Vietnam, 300 m, 30 April-1 May 2005, KT Park and S Kim, slide gen. no. SNU-9281/S Kim.

\section{Distribution}

Oriental: Vietnam (North), Indonesia (Northeast, West; Lvovsky 2000a).

Promalactis reniformis Kim and Park, sp. nov.

(Figures 1Q, R, 2I, and 4Q-S) 


\section{Diagnosis}

This species is superficially close to Promalactis tricuspidata Wang and $\mathrm{Li}$ in the wing pattern, but can be easily distinguished by the female genitalia with a large, reniform lamella postvaginalis, deeply incised at middle.

\section{Description}

Adult (Figure 1Q, R). Head (Figure 2I): Frons and vertex white mixed with greyishbrown. Scape of antenna entirely white, except dark brown apex dorsally, as long as diameter of eye; flagellum white and dark brown alternately. Second segment of labial palpus dark brown dorsally, pale brown ventrally, gradually darker to apex, longer than third segment; third segment dark brown, except white at basal and apical parts. Thorax: Thorax and tegula brown dorsally. Wing expanse 7.0-7.5 mm. Forewing ground colour yellowish-brown; white markings irregularly shaped and rarely surrounded by fuscous scales: one band-like, near at base; two spots connected at middle of one-third forewing; second band-like one, short and oblique from onethird posterior margin; third band-like one, short and oblique from one-half posterior margin, connecting to the other short band-like one, extending to before the tornus by fuscous scales; one reniform costal patch at two-thirds of costa; two apical spots surrounded by fuscous scales: smaller one at vein radius VI and large one at apex; fringes yellow from two-thirds of costal margin to two-thirds of outer margin. Hindwing lanceolate; fringes greyish-brown. Leg: hind tibia pale yellowish-brown densed with pale greyish-brown fringes; tarsus pale yellowish-brown, except white at basal parts of fifth and fourth segments.

\section{Male genitalia. Unknown.}

Female genitalia (Figure 4Q-S). Apophysis posterioris more than 1.5 times length of apophysis anterioris. Lamella postvaginalis large, reniform, incised deeply, setose on caudal margin with numerous orbicular sclerites laterally. Lamella antevaginalis small and trapezoid-shaped. Antrum hexagonal-like. Ductus bursae wide at base, membranous, gradually narrowed to one-third, then swollen forming a balloon-like part from one-third. Corpus bursae ovate; signum absent.

\section{Holotype}

One female, Cuc Phuong Nat. Park, Vietnam, 196 m, 3-4 July 2008, JB Heppner, slide gen. no. SNU-9039/S Kim.

\section{Etymology}

The specific name of the new species is derived from the Latin, reni (=kidney) plus forma (form $-i s)$ (= form), referring to lamella postvaginalis reniform of female genitalia. 
1344 S. Kim et al.

\section{Distribution}

Oriental: Vietnam (North).

Promalactis tauricournis Du et al., 2011

(Figures 1S, T, 2J, and 4T, U)

Promalactis tauricournis Du et al., 2011, Zootaxa, 3044: 49-64.

\section{Diagnosis}

This species is superficially close to $P$. asperiola sp. nov. in the wing pattern, but it can be differentiated by the female genitalia with sclerotized, cylindrical ductus bursae and the wrinkled seventh sternite laterally.

\section{Material examined}

One female, Tam Dao Nat. Park, Vietnam, 1108 m, 16-19 June 2008, JB Heppner, slide gen. no. SNU-9048/S Kim.

\section{Remark}

According to the original description (Du et al. 2011) of the species, the forewing has white scales mixed with the fuscous scales before the tornus, but the Vietnamese specimen has no such white scales. A further study is needed when additional specimens are available.

\section{Distribution}

Oriental: Vietnam (North), China (South; Du et al. 2011).

Promalactis tricuspidata Wang and Li, 2004

(Figures 1U, V, 2K, and 3G, H)

Promalactis tricuspidata Wang and Li, 2004, Oriental. Insects, 38: 2.

\section{Diagnosis}

This species externally similar to $P$. reniformis sp. nov. in the wing pattern, but it is differentiated by the trapezoidal antrum and the short ductus bursae in female genitalia.

\section{Material examined}

Three males, Cuc Phuong Nat. Park, Vietnam, 200 m, 1-2 July 2008, JB Heppner, slide gen. no. 9051, 9040, 9278/S Kim; one male, same locality, 155 m, 4-7 May 2009, JB Heppner, slide gen. no. SNU-9333/S Kim. 


\section{Distribution}

Oriental: Vietnam (North), China (Southwest; Wang 2006).

\section{Acknowledgements}

We are grateful to Dr Thomas C. Emmel and McGuire Center for Lepidoptera and Biodiversity, Florida Museum of Natural History, University of Florida, USA, for laboratory support, and Dr Ulf Eischberger (Marktleuthern, Germany) for research grant during the first author's visiting period from December 2011 to January 2012. We are also indebted to Vuong Pham and Cuong Ngyuen, National Institute of Plant Protection, Hanoi, for their helpful supports in collecting of material.

\section{References}

Du ZH, Li HH, Wang SX. 2011. Taxonomic study of the genus Promalactis Meyrick (Lepidoptera, Oecophoridae) from Hainan Province, Chain. Zootaxa. 3044:49-64.

Du ZH, Zhang L, Wang SX. 2009. Four new species of the genus Promalactis Meyrick 1908 from China (Lepidoptera: Oecophoridae), SHILAP Rev. Lepidopterol. 37:319-325.

Fujisawa K. 2002. The genus Promalactis (Oecophoridae) from Japan. Jpn Heterocer J. 218:337-350.

Kim S, Park KT, Byun BK, Heppner JB, Lee S. 2012. Genus Promalactis (Lepidoptera, Oecophoridae) from Northern Vietnam, Part II: Six new species of the genus. J Nat Hist. 46:897-909.

Kim S, Park KT, Byun BK, Lee S. 2010. Genus Promalactis (Lepidoptera, Oecophoridae) from Northern Vietnam, Part I: Descriptions of five new species. Fla Entomol. 93:546-557.

Kornerup A, Wanscher JH. 1978. Methuen handbook of colour. 3rd ed. London: Methuen; $252 \mathrm{pp}$.

Lvovsky AL. 1976. Some little known species of Far Eastern Oecophorids (Lepidoptera: Oecophoridae). Trudy Zool Inst Leningrad. 67:56-59.

Lvovsky AL. 1985. New species of the broad winged moths (Lepidoptera, Oecophoridae) from Primorye region. Trudy Zool Inst Leningrad. 134:95-101.

Lvovsky AL. 1986. A review of the broad-winged moths (Lepidoptera, Oecophoridae) of the Far East. Trudy Zool Inst Leningrad. 145:72-74.

Lvovsky AL. 1988. New and little-known species of broad-winged moths (Lepidoptera, Oecophoridae) from Vietnam. Trudy Zool Inst Akad Nauk SSSR. 176:120-128.

Lvovsky AL. 1997. New and little-known species of oecophorid moths (Lepidoptera, Oecophoridae) from Vietnam. Zool Zh. 76:759-762.

Lvovsky AL. 2000a. New and little known species of oecophorid moths of the genera Epicalima Dyar, 1903 and Promalactis Meyrick, 1908 (Lepidoptera, Oecophoridae) from South East Asia. Entomol Obozr. 79:664-691.

Lvovsky AL. 2000b. A new subspecies of Promalactis autoclina Meyrick, 1935 from Indonesia (Lepidoptera, Oecophoridae). Würzburg. Atalanta. 31:245-247.

Lvovsky AL. 2007. New species of the moth genus Promalactis Meyrick, 1908 from Indonesia and Vietnam (Lepidoptera: Oecophoridae). Zoosystem Ross. 16:127-130.

Meyrick E. 1908a. Descriptions of Indian micro-lepidoptera. J Bombay Nat Hist Soc. $15: 806-812$.

Meyrick E. 1908b. New micro-lepidoptera from India and Burma. Records Indian Mus. 2:395-400.

Park KT. 1981. A revision of the Genus Promalactis of Korea (Lepidoptera, Oecophoridae). Korean J Plant Protect. 20:43-50. 


\section{S. Kim et al.}

Park KT, Park YM. 1998. Genus Promalactis Meyrick (Lepidoptera, Oecophoridae) from Korea, with descriptions of six new species. J Asia Pacific Entomol. 1:51-70.

Wang SX. 2006. Oecophoridae of China (Insecta, Lepidoptera). Beijing: Science Press; 258 pp.

Wang SX, Kendrick RC, Sterling P. 2009. Microlepidoptera of Hong Kong: Oecophoridae I: the genus Promalactis Meyrick. Zootaxa. 2239:31-44. 